\title{
The Influence of Liquid Surface Segregation on the Pitting Corrosion Behavior of Semi-Solid Metal High Pressure Die Cast Alloy F357
}

\author{
H. Möller ${ }^{*}$ and E.P. Masuku \\ CSIR, P.O. Box 395, Pretoria, 0001, South Africa
}

\begin{abstract}
Semi-solid metal processing results in liquid segregation at the surface of the components. The pitting behavior of this surface layer of semi-solid metal processed alloy F357 was compared with the centre (or bulk) of cast plates in $3.5 \% \mathrm{NaCl}$ aqueous solution. It is shown that pitting attack occurs preferentially in the eutectic regions at the interface between silicon particles and the alpha phase in the eutectic. Since the surface liquid segregation layer consists of mainly eutectic, this makes semi-solid metal processed components specially susceptible to pitting corrosion attack.
\end{abstract}

Keywords: Semi-solid metal (SSM) processing, pitting corrosion, surface liquid segregation.

\section{INTRODUCTION}

Semi-solid metal (SSM) processing is a unique manufacturing method to produce near-net shape products for various industrial applications [1]. The aim is to obtain a semi-solid structure (which is free of dendrites) with the solid present in a near spherical form. This semi-solid mixture flows homogeneously, behaving as a thixotropic fluid with viscosity depending on the shear rate and fraction of solid [2]. There are two main different SSM processing methods namely rheocasting and thixocasting. With thixocasting, a specially prepared billet of solid material with a globular microstructure is reheated into the semi-solid range, followed by a forming process such as high pressure die casting (HPDC). Rheocasting on the other hand involves preparation of the SSM slurry directly from the liquid, followed by HPDC. Rheocasting has become the preferred semi-solid process, due to the higher costs that are associated with thixocasting [2].

The nature of the SSM slurry (i.e. solid spherical grains suspended in liquid), makes it prone to liquid segregation during the forming process. There have been a number of reported studies investigating the modeling and quantifying typical segregation phenomena in SSM forming [3-7]. These articles mainly focused on bulk segregation to establish appropriate die design methodology. It was demonstrated that die design had a significant impact on the degree and location of liquid segregation. Regions of high strain rate were found to have a higher liquid fraction [5]. It has been shown by one of the authors before, that in SSM-HPDC plates, a surface liquid segregation (SLS) phenomenon existed [8]. The SLS layer (eutectic layer) varied in thickness of between 0.7 and $1.1 \mathrm{~mm}$ (using the upper limit specification for $\mathrm{Si}$ of $7.5 \%$ as the criteria for determining the thickness based on OES analysis [8]). It was postulated that the surface segregation phenomenon that was observed is a characteristic of SSM forming and that the degree of segregation will be dependent on alloy composition, die design and processing parameters [8].

*Address correspondence to this author at the CSIR, P.O. Box 395, Pretoria, 0001, South Africa; E-mail: hmoller@csir.co.za
The pitting corrosion behavior of semi-solid metal processed A356/7 has been studied before by Bastidas and co-workers [9], as well as Tahamtam and Boostani [10]. Park and co-workers [11] compared the corrosion properties of rheocast and low-pressure cast A356. In none of these papers was the influence of a possible SLS layer considered. In fact, preparation of the corrosion samples involved grinding and polishing of the sample surfaces, resulting in removal of any SLS layers. Corrosion is a surface phenomenon, and it has been shown that the surface and the bulk of SSM-processed components differ vastly. Therefore, the purpose of this paper is to compare the corrosion behavior of the SLS layers and the bulk of SSM-HPDC plates of alloy F357 in chloride medium.

\section{MATERIALS AND METHODOLOGY}

Semi-solid metal slurries of alloy F357 (chemical composition of the melt given in Table 1) were prepared using the CSIR rheocasting process [12]. Note that alloy F357 is the Be-free version of alloy A357. Plates $(4 \mathrm{~mm} \times 80 \mathrm{~mm} \times 100 \mathrm{~mm})$ were cast in steel moulds with a 130 ton HPDC machine. The surface chemical composition was measured using a Thermo Quantris Optical Emission Spectrometer (OES) which had been calibrated for Al-Si alloys. The depth profile was determined by first measuring the chemical composition of the as cast surface. Material was then removed by a grinding process followed by measurement of the amount of material removed and chemical analysis. Chemical profiles of the main alloying elements were plotted for the cross-section of the plate. A more detailed investigation of the chemical composition was performed in a previous paper by one of the authors [8].

Table 1. Chemical Composition of the Melt (wt\%) Used to Produce SSM-HPDC Alloy F357

\begin{tabular}{|c|c|c|c|c|c|c|c|c|}
\hline Al & Si & Mg & Fe & $\mathbf{C u}$ & Mn & Zn & Ti & Sr \\
\hline \hline Bal. & 7.11 & 0.51 & 0.08 & 0.01 & 0.01 & 0.01 & 0.16 & 0.02 \\
\hline
\end{tabular}

Metallographic samples of cross-sections of the casting were prepared and evaluated using an optical metallurgical microscope. 
Polarization measurements were carried out after immersion of the samples for 30 minutes in $3.5 \% \mathrm{NaCl}$ aqueous solution. A Solartron 1287 electrochemical interface was used with a scan rate of $1 \mathrm{mV} / \mathrm{s}$. Repeatability was tested by making at least three different scans on different areas on the samples. A conventional three-electrode configuration was used with two graphite rod counter electrodes (diameter $=8 \mathrm{~mm}$ and depth into electrolyte $=60 \mathrm{~mm}$ ) on either side of the working electrode. The surface area of the counter electrodes (combined area $=30 \mathrm{~cm}^{2}$ ) was significantly larger than that of the $1.54 \mathrm{~cm}^{2}$ working electrode. A silver-silver chloride (SSC) reference electrode (with saturated $\mathrm{KCl}$ filling solution) was employed. To study the "bulk samples" (beneath the SLS layer) the samples were hand polished with different grades of emery paper (down to grade 1200). To study the SLS layer, only a few seconds polishing with grade 1200 was used in order not to remove the layer. In all samples, this was followed by diamond paste polishing down to $1 \mu \mathrm{m}$. Final polishing was done using a colloidal silica solution. Optical micrographs were taken before polarisation to ensure that the SLS layer had not been removed for the "SLS" samples and that it had been removed for the "bulk" samples.

\section{RESULTS AND DISCUSSION}

Chemical Analysis. Chemical analysis depth profiles of the main alloying elements determined using the OES are shown in Fig. (1). Only the results from one side of the plate are shown, as it has been proven before that the effect is similar on both sides of the plates [8]. The surface $\mathrm{Si}$ and $\mathrm{Mg}$ contents are relatively high, while the Ti content is relatively low. The eutectic composition of binary Al-Si alloys is approximately $12 \%$ [13], which is very close to what has been measured at the surface of these plates. The Si content drops significantly towards the centre of the plate. Si and $\mathrm{Mg}$ are alloying elements that would be found in the eutectic of this alloy, hence indicating that the regions with high content of these alloying elements were regions of high liquid content. The Ti content displays opposite behavior - $\mathrm{Ti}$ is a grain refiner for the primary $\alpha$ phase and would therefore be present where the $\alpha$ grains would be of highest density.

Microstructures. Microstructural analyses of crosssections of the castings (Fig. 2) reveal the presence of the SLS layer. The region close to the surface was composed of mainly eutectic, while closer to the centre of the casting, microconstituents were mainly globular $\alpha$ primary grains and smaller amounts of eutectic.

Corrosion Behavior. Aluminum alloys are susceptible to pitting corrosion in solutions containing halide ions, of which $\mathrm{Cl}^{-}$is the most common [10]. Chloride ions result in the localized breakdown of the passive film, which in turn leads to the formation of corrosion pits. Fig. (3) shows typical polarization curves for SSM-HPDC alloy $\mathrm{F} 357$ in $3.5 \% \mathrm{NaCl}$ aqueous solution for the SLS layer and the bulk of the plates.

The cathodic curve for the SLS layer shows a plateau, which suggests reduction of dissolved oxygen (cathodic Tafel constant $\left.\beta_{\mathrm{c}} \rightarrow \infty\right)$. After the plateau, the corrosion potential is found $\left(\mathrm{E}_{\mathrm{corr}}=-0.76 \mathrm{~V}_{\mathrm{SSC}}\right)$, and immediately thereafter the pitting potential $\left(\mathrm{E}_{\mathrm{Pit}}\right)$. The anodic curve therefore does not show a passive plateau. The polarization behavior of the SLS layer was found to be reproducible. This (a)

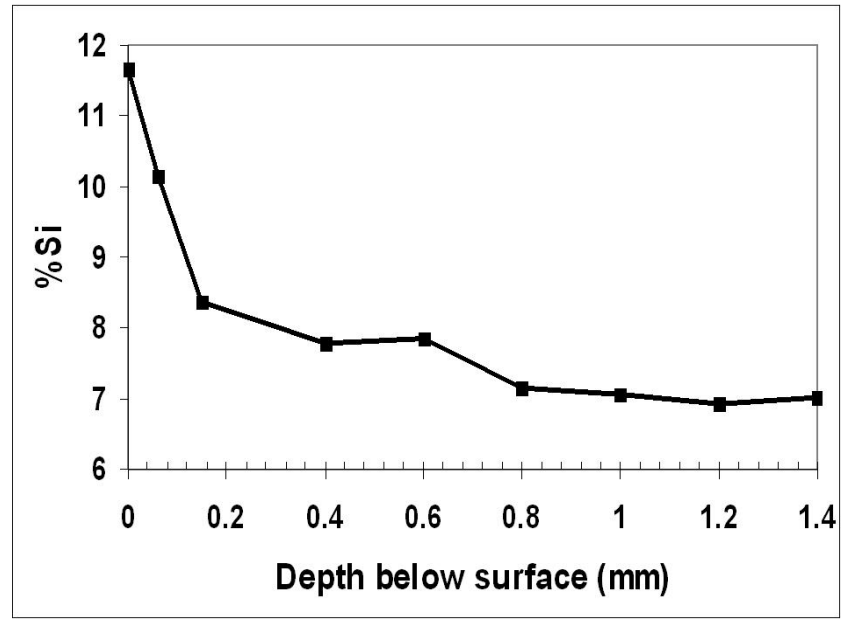

(b)

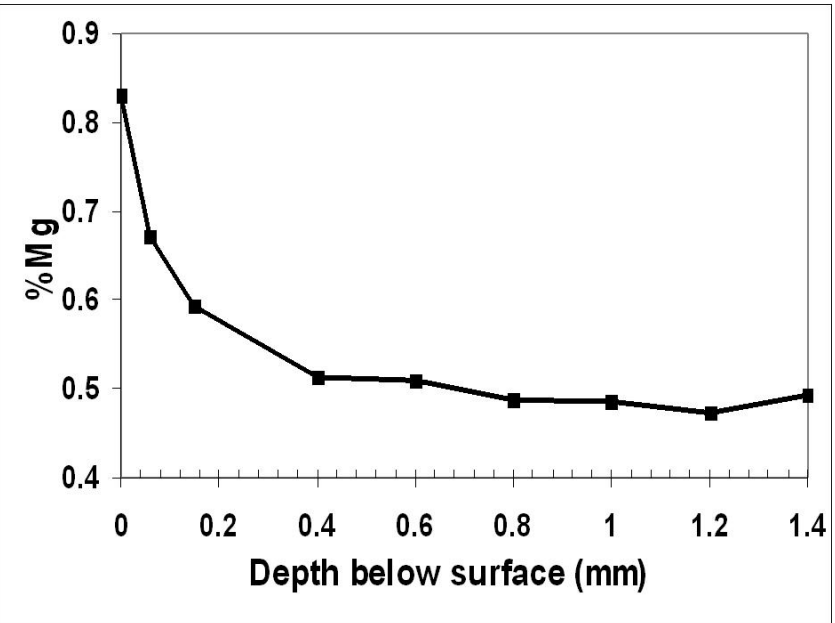

(c)

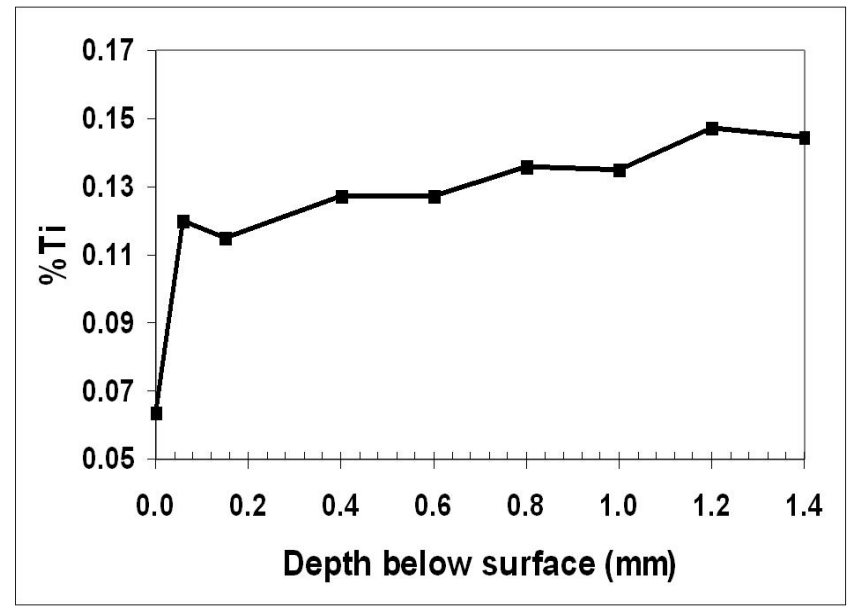

Fig. (1). OES chemical analyses depth profiles for a) $\mathrm{Si}, \mathbf{b}) \mathrm{Mg}$ and c) $\mathrm{Ti}$.

is to be expected as the layer consists of mainly eutectic and no heterogeneity in composition or microstructure is found (Fig. 2). However, two types of polarization curves were found for the bulk of the sample. The first type (called "Bulk 
1") displayed a corrosion potential of $\mathrm{E}_{\text {corr }}=-0.82 \mathrm{~V}_{\mathrm{SSC}}$ and immediately thereafter the pitting potential without a passive plateau (similar to the SLS layer). This corresponds well to the results presented by Bastidas and co-workers [9]. The rate at which the anodic currents increase for "Bulk 1" is, however, significantly lower than for the SLS layer (with anodic Tafel constants $\beta_{\mathrm{a}}$ equal to $0.036 \mathrm{~V}$ and $0.024 \mathrm{~V}$ for "Bulk 1 " and the SLS layer respectively). The second type of polarization behavior for the bulk of the plate (called "Bulk 2") shows a significantly less-noble $\mathrm{E}_{\text {corr }}=-0.90 \mathrm{~V}_{\mathrm{SSC}}$. In this case, the alloy displays more passive behavior (with anodic Tafel constant $\beta_{\mathrm{a}}=$ $0.16 \mathrm{~V}$ ), followed by a pitting potential of $\mathrm{E}_{\mathrm{Pit}}=-0.74 \mathrm{~V}_{\mathrm{SSC}}$.

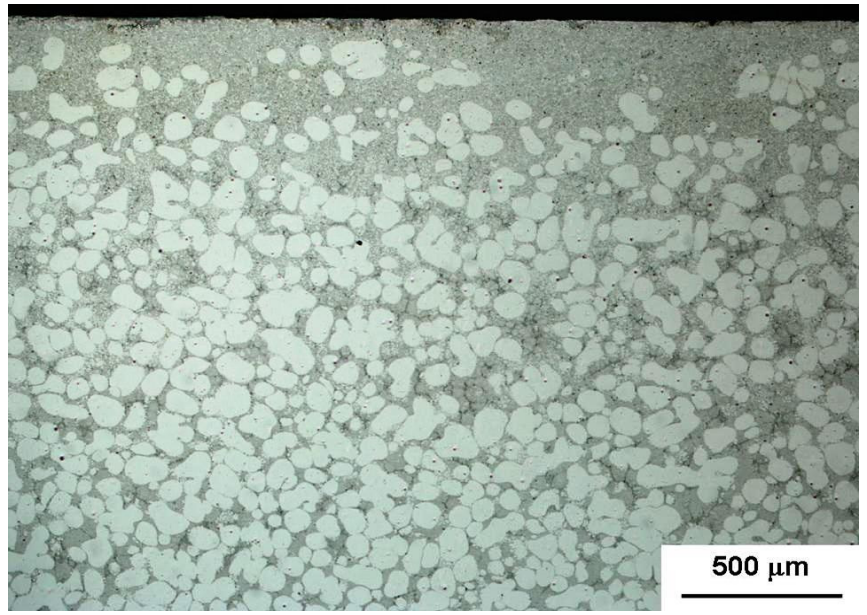

Fig. (2). Optical micrograph of a cross-section of a SSM-HPDC F357 plate.

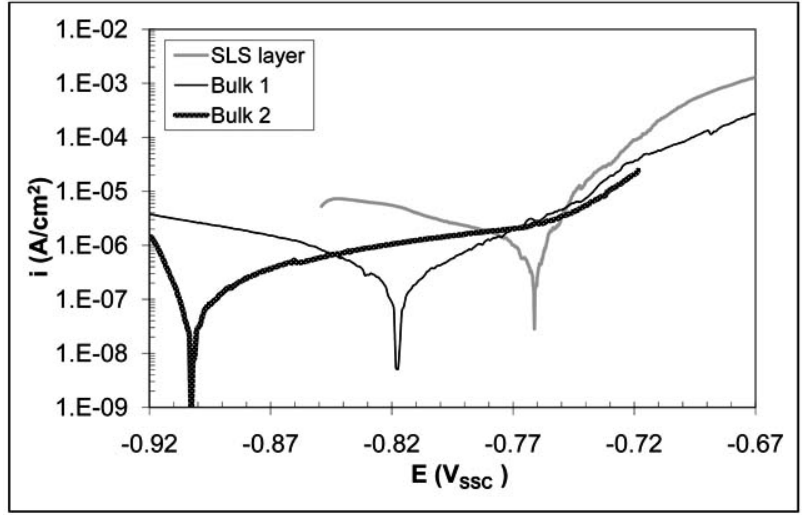

Fig. (3). Typical polarisation curves for the SLS layer and the bulk of SSM-HPDC F357 plates in $3.5 \% \mathrm{NaCl}$ aqueous solution after 30 minutes immersion. Scan rate was $1 \mathrm{mV} / \mathrm{s}$.

To explain these observations, Figs. $(\mathbf{2}, \mathbf{4}, \mathbf{5})$ must be considered. Figs. $(\mathbf{2}, \mathbf{4 a}, \mathbf{4 b})$ show that the surface layer is composed of mainly eutectic, while the bulk of the casting (Figs. $\mathbf{4 c}, \mathbf{4 d}, \mathbf{5}$ ) consists of mainly globular $\alpha$ primary grains and smaller amounts of eutectic. The more noble corrosion potential of the SLS layer is due to the higher area fraction of the more noble silicon particles compared to the bulk, where more of the less-noble $\alpha$-aluminium grains are found. The two different behaviours observed for the bulk of the plates give an indication of the heterogeneity of the microstructure that is obtained by means of SSM-HPDC - this is illustrated (a)

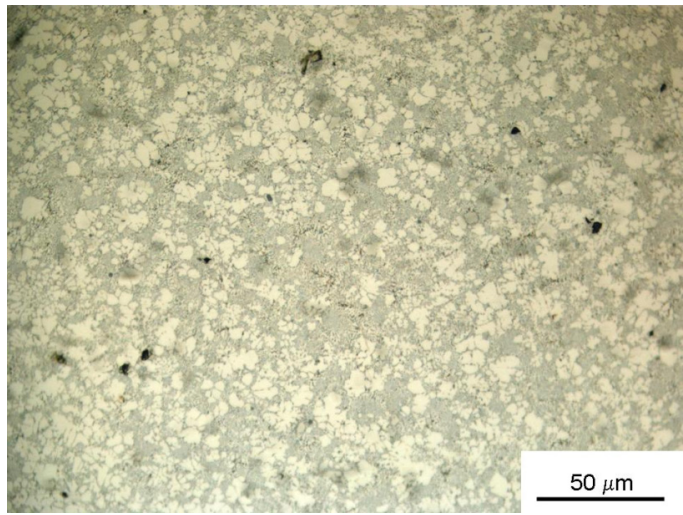

(b)

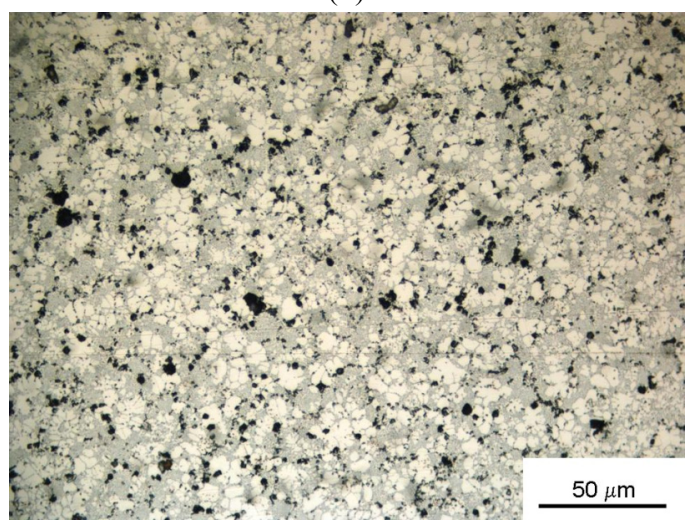

(c)

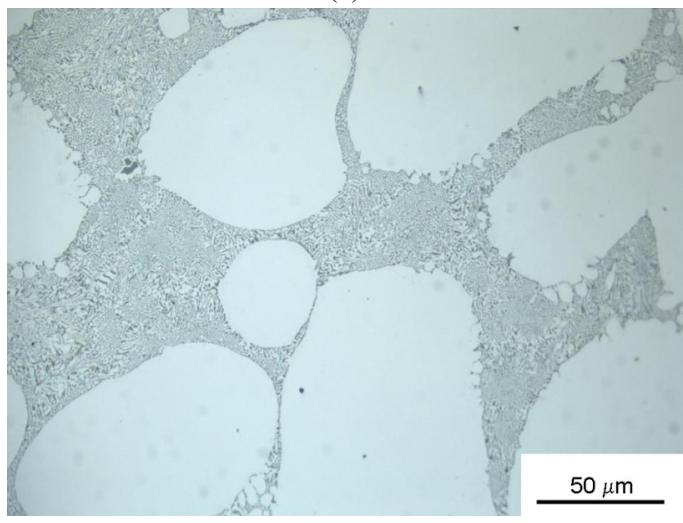

(d)

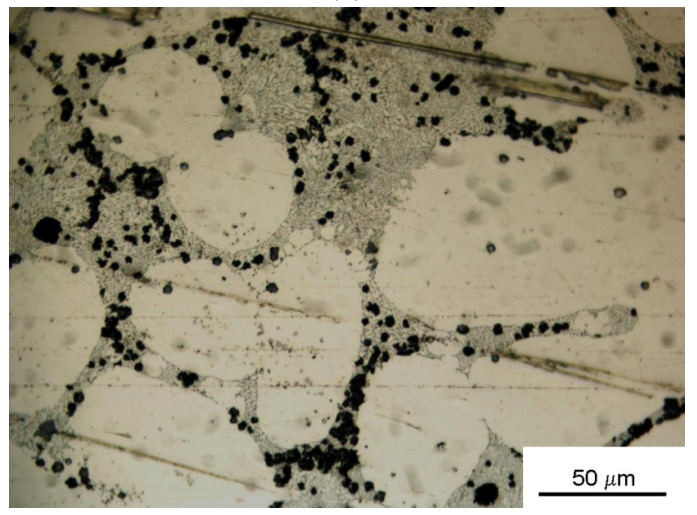

Fig. (4). Optical micrographs of the SLS layer a) before polarization and b) after polarization and for the bulk c) before polarization and $\mathbf{d}$ ) after polarization. 
graphically in Fig. (5). It is seen from Fig. (5a) that the area corresponding to "Bulk 2" contains more primary $\alpha$-Al than the area in Fig. (5b) corresponding to "Bulk 1". Image analysis of Fig. (5) revealed area fractions of primary $\alpha-\mathrm{Al}$ of 0.63 and 0.48 for Fig. $(\mathbf{5 a}, \mathbf{5 b})$ respectively. The presence of more $\alpha-\mathrm{Al}$ (and less eutectic) in "Bulk 2" (Fig. 5a) results in a less-noble corrosion potential, because primary alpha particles are almost $100 \%$ pure Al [11]). Energy dispersive spectroscopy (EDS) using a Scanning Electron Microscope (SEM) showed that the $\alpha-\mathrm{Al}$ contains approximately $1.2 \% \mathrm{Si}$ as major alloying element, confirming that $\alpha-\mathrm{Al}$ is almost pure $\mathrm{Al}$ [11]. The maximum solid solubility of $\mathrm{Si}$ in $\mathrm{Al}$ in binary Al-Si alloys is $1.6 \%$ at $577^{\circ} \mathrm{C}$ [13].

(a)

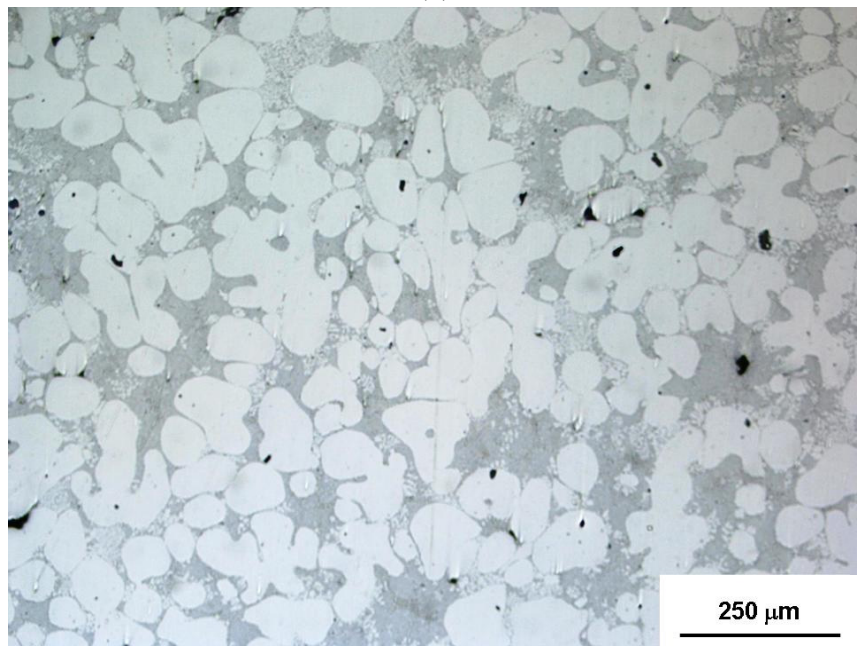

(b)

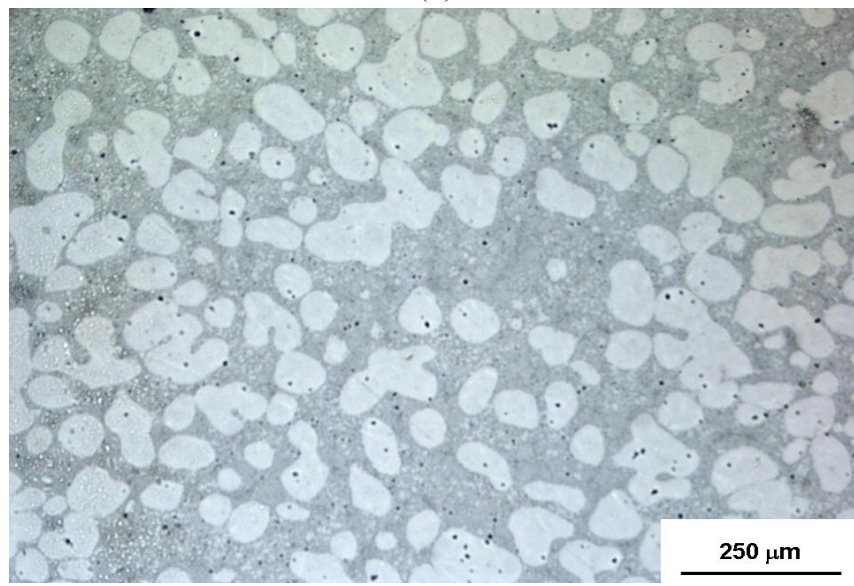

Fig. (5). Optical micrographs of different areas that are observed in the bulk of the sample a) area corresponding to "Bulk 2" polarization curve and b) area corresponding to "Bulk 1" polarization curve.
Please note that areas corresponding to both "Bulk 1" and "Bulk 2" could be found at different spots on the same plates cast by SSM-HPDC. However, the behaviour represented by "Bulk 1" was most common. This heterogeneity in the microstructure in the bulk of the sample is due to the unique flow behaviour that is achieved with SSM-HPDC. Optimisation of dies and casting parameters should be able to minimise this heterogeneity, but is not the focus of the current paper.

Furthermore, from Fig. (4b, 4d) it can also be seen that the pitting attack is favoured through the eutectic regions, while the primary $\alpha$-Al grains were mostly unattacked. The corrosion attack occurs predominantly around the silicon particles, which act as local cathodes relative to the eutectic aluminium phase [9-11]. The SLS layer consists of mainly eutectic, making it more susceptible to pitting corrosion than the bulk of the sample. The higher anodic Tafel constant of "Bulk 1" compared to the SLS layer is most likely due to the presence of the corrosion resistant primary $\alpha$-Al grains, which decreases the total interface area between silicon and $\alpha$ phase in the eutectic. There are therefore less possible pitting initiation sites in "Bulk 1" than in the SLS layer. This effect is more significant in "Bulk 2" (with even more primary $\alpha$-Al grains and therefore less possible pitting initiation sites than "Bulk 1" - see Fig. 5) that it displays more passivity before the pitting potential is reached.

A summary of a Tafel analysis of the polarisation curves is presented in Table 2 . In this analysis, the corrosion potentials $\left(\mathrm{E}_{\mathrm{corr}}\right)$, pitting potentials $\left(\mathrm{E}_{\mathrm{pit}}\right)$, corrosion current densities ( $i_{\text {corr }}$, determined through Tafel extrapolation to the corrosion potential $)$, anodic and cathodic Tafel constants $\left(\beta_{\mathrm{a}}\right.$ and $\left.\beta_{c}\right)$ and the polarisation resistances were determined $\left(\mathrm{R}_{\mathrm{p}}\right)$.

Finally, cross sections of the samples after polarisation were made to view the pitting attack from side-on. These results are shown in Fig. (6). The preferential attack through the eutectic regions and the high corrosion resistance of the primary $\alpha$-Al can be seen. The severe susceptibility of the SLS layer to pitting attack is also evident.

\section{CONCLUSIONS}

Pitting corrosion of Al-Si-Mg alloy F357 occurs preferentially in the eutectic regions, whereas the primary $\alpha$ Al grains remain corrosion resistant. Semi-solid metal processing causes surface liquid segregation, with the surface layer consisting of mainly eutectic. This makes semisolid metal processed components susceptible to pitting corrosion and needs to be considered whenever such components might be exposed to chloride media.

Table 2. Tafel Analysis of the Polarisation Curves in Fig. (3)

\begin{tabular}{|c|c|c|c|c|c|c|}
\hline & $\mathbf{E}_{\text {corr }}\left(\mathbf{V}_{\text {ssc }}\right)$ & $\mathbf{E}_{\text {pit }}\left(\mathbf{V}_{\mathbf{s s c}}\right)$ & $\mathbf{i}_{\text {corr }}\left(\mathbf{A} / \mathbf{c m}^{2}\right)$ & $\boldsymbol{\beta}_{\mathbf{a}}\left(\mathbf{a t} \mathbf{E}_{\text {corr }}\right)$ & $\boldsymbol{\beta}_{\mathbf{c}}\left(\mathbf{a t} \mathbf{E}_{\text {corr }}\right)$ & $\mathbf{R}_{\mathbf{p}}\left(\mathbf{\Omega} . \mathbf{c m}^{2}\right)$ \\
\hline \hline SLS layer & -0.76 & -0.76 & $4 \times 10^{-6}$ & 0.024 & $\infty$ & $2.6 \times 10^{3}$ \\
\hline Bulk 1 & -0.82 & -0.82 & $7 \times 10^{-7}$ & 0.036 & $\infty$ & $2.2 \times 10^{4}$ \\
\hline Bulk 2 & -0.90 & -0.74 & $3 \times 10^{-7}$ & 0.16 & $\infty$ & $2.3 \times 10^{5}$ \\
\hline
\end{tabular}


(a)

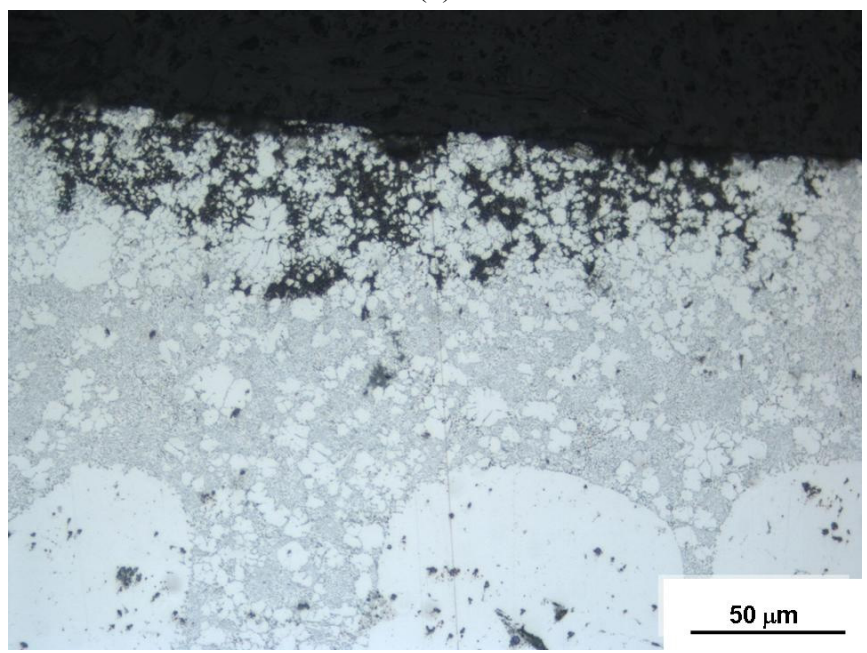

(b)

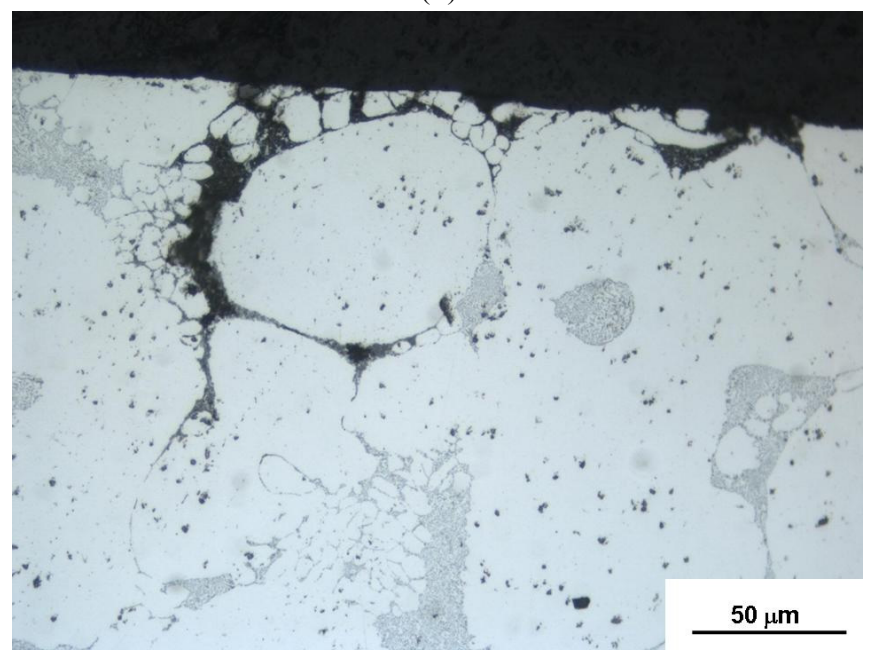

Fig. (6). Optical micrographs of cross sections of a) the SLS layer and $\mathbf{b}$ ) the bulk after polarization in $3.5 \%$ aqueous $\mathrm{NaCl}$ solution at $-0.70 \mathrm{~V}_{\mathrm{SSC}}$ for 15 minutes..

\section{ACKNOWLEDGEMENTS}

The authors would like to thank the Department of Materials Science and Metallurgical Engineering, University of Pretoria, South Africa, for making their electrochemical testing equipment available.

\section{REFERENCES}

[1] Atkinson HV. Modelling the semisolid processing of metallic alloys. Prog Mater Sci 2005; 50: 341-412.

[2] Flemings MC, Martinez RA. Principles of microstructural formation in semi-solid metal processing. Solid State Phenom 2006; 116-117: 1-8.

[3] Noll T, Friedrich B, Hufschmidt M, Modigell M, Nohn B Hartmann D. Evaluation and modelling of chemical segregation effects for thixoforming process. Adv Eng Mater 2003; 5: 156-60.

[4] Kang CG, Jung HK. A study on solutions for avoiding liquid segregation phenomena in thixoforming process: Part I: constitutive modelling and finite element method simulations for die design. Met Mater Trans B 2001; 32B: 119-27.

[5] Kang CG, Jung HK. A study on solutions for avoiding liquid segregation phenomena in thixoforming process: Part II: net shape manufacturing of automotive scroll component. Met Mater Trans B 2001; 32B: 129-36.

[6] Hufschmidt M, Modigell M, Petera J. Modelling and simulation of forming processes of metallic suspensions under non-isothermal conditions. J Non- Newtonian Fluid Mech 2006; 134: 16-26.

[7] Seo PK, Kim DU, Kang CG. The effect of the gate shape on the microstructural characteristic of the grain size of Al-Si alloy in the semi-solid die casting process. Mater Sci Eng A 2007; 445-446: 20-30.

[8] Govender G, Möller H. Evaluation of surface chemical segregation of semi-solid cast aluminium alloys. Solid State Phenom 2008; 141-143: 433-8.

[9] Bastidas JM, Forn A, Baile MT, Polo JL, Torres CL. Pitting corrosion of A357 alumium alloy obtained by semisolid processing. Mater Corrosion 2001; 52: 691-6.

[10] Tahamtan S, Boostani AF. Qualitative analysis of pitting corrosion behaviour of thixoformed A356 alloy in chloride medium using electrochemical techniques. Mater Des 2009; 30: 2483-2489.

[11] Park C, Kim S, Kwon Y, Lee Y, Lee J. Mechanical and corrosion properties of rheocast and low -pressure cast A356-T6 alloy. Mater Sci Eng A 2005; 391: 86-94.

[12] Ivanchev L, Wilkins D, Govender G. In: Proceedings of the 8th International Conference on Semi-solid processing of alloys and composites; September 2004; WPI's Metal Processing Institute, Limassol: Cyprus 2004.

[13] Murray JL, McAlister AJ. The Al-Si system. J Phase Equilib 1984; 5: 74-84.

(C) Möller and Masuku; Licensee Bentham Open.

This is an open access article licensed under the terms of the Creative Commons Attribution Non-Commercial License (http://creativecommons.org/licenses/by$\mathrm{nc} / 3.0 /$ ) which permits unrestricted, non-commercial use, distribution and reproduction in any medium, provided the work is properly cited. 